\title{
Urgences
}

\section{Le salut de la chair et de la poésie dans Le ciel de Québec de Jacques Ferron}

\section{Andrée Mercier}

Numéro 28, mai 1990

Le roman comme poétique

URI : https://id.erudit.org/iderudit/025585ar

DOI : https://doi.org/10.7202/025585ar

Aller au sommaire du numéro

Éditeur(s)

Urgences

ISSN

0226-9554 (imprimé)

1927-3924 (numérique)

Découvrir la revue

Citer cet article

Mercier, A. (1990). Le salut de la chair et de la poésie dans Le ciel de Québec de Jacques Ferron. Urgences, (28), 20-29. https://doi.org/10.7202/025585ar d'utilisation que vous pouvez consulter en ligne.

https://apropos.erudit.org/fr/usagers/politique-dutilisation/ 


\section{Le salut de la chair et de la poésie dans Le ciel de Québec de Jacques Ferron}

Andrée Mercier

Pour ma part je ne crois pas a l'écrivain conçu comme héros. L'important est d'avoir une littérature déconcertante poussant sur une langue vivante. Cette vie, ce sont les poètes qui l'entretiennent.

Jacques Ferron, Escarmouches, tome 2, p. 64.

Rechercher quel discours Le ciel de Québec pourrait tenir sur la poésie semble sans doute, du moins pour les lecteurs ayant le roman de Ferron à l'esprit, une entreprise inutile. Le portrait féroce du poète Saint-Denys Garneau, dit Orphée, constitue, en effet, une charge assez claire à l'endroit d'une poésie trop intimiste, détachée de la réalité sociale, sans qu'il soit nécessaire d'en rajouter. Vive repartie à Chronique de l'âge amer de Robert Charbonneau qui, un peu plus tôt, avait contribué à relancer l'intérêt pour l'auteur de Regards et jeux dans l'espace, Le ciel de Québec entend clairement, quant à lui, crever la « boursouflure de renommée » dont jouit, à tort, le poète ${ }^{1}$. Le procès de Saint-Denys Garneau étant celui de toute poésie exotique ou métaphysique indifférente aux problèmes immédiats du monde, on pourrait en conclure, dès lors, que le roman de Ferron n'admet en retour qu'une poésie enracinée et patriotique, qu'un repérage exhaustif des sources et auteurs utilisés (cités ou non) permettrait d'étoffer davantage.

S'en tenir à cette première constatation, cependant, serait confondre l'éventuelle signification d'un texte avec ce que son narrateur (ou ses principaux personnages) énonce et ignorer, de ce fait, le rôle de la structure même du roman dans la production

1 Le ciel de Québec a été publié pour la première fois en 1969, soit deux ans après Chronique de l'age amer de Charbonneau. Nous citerons, toutefois, l'édition de 1979 parue chez VLB éditeur. Les numéros de pages indiqués entre parenthèses renverront donc au Ciel de Québec et à cette édition. 
du sens. En d'autres mots, si les propos du narrateur tendent à rejeter Saint-Denys Garneau et sa poésie, il y a tout de même, au niveau fictionnel, intégration et récupération de l'écrivain. Saint-Denys Garneau constitue, en effet, un personnage du Ciel de Québec et participe pleinement, à ce titre, à l'organisation narrative et figurative du texte. La trame actorielle du roman, rappelons-le, intègre, en plus du poète, membres du clergé catholique et de l'Eglise anglicane, politiciens de tout acabit, Amérindiens, Métis, écrivains et artistes, Québécois d'origine française, irlandaise et écossaise, etc., soit plus de deux cents personnages, desquels une vingtaine seulement - dont Orphée - occupe le premier plan; pendant que la trame événementielle entremêle, de son côté, toute une série d'incidents, tels la fondation d'une nouvelle paroisse, l'k enquébecquoisement " du pasteur Frank-Anacharcis Scot, les petites manigances des politiciens de Québec et d'Ottawa et la descente aux enfers du poète ${ }^{2}$... Pour être saisie le plus justement possible, il apparaît donc nécessaire que la figure d'Orphée soit intégrée et mesurée à l'ensemble de la fiction et non pas maintenue aux propos et commentaires du narrateur (regroupés surtout dans les chapitres XX, XXI et XXII d'un roman qui en compte trente-cinq, une fois inclus le chapitre de conclusion). À supposer que Le ciel de Québec développe, par l'intermédiaire d'Orphée, une vision et une critique de la poésie, c'est en replaçant la figure du poète dans la structure fictionnelle du texte et, autant que possible, dans sa progression narrative, que l'on risque encore de l'atteindre le mieux.

Si la place "matérielle» occupée par le personnage d'Orphée dans la fiction démontre déjà l'importance d'une réflexion poétique dans Le ciel de Québec, ce discours, toutefois, ne tient pas tout entier dans le personnage de l'écrivain: la figure de l'écriture poétique se trouve également

2 Dans son article sur Le ciel de Québec, Alonzo Le Blanc propose ce résumé du roman: «Pendant que les politiciens s'amusent et que les bourgeois s'interrogent, pendant que les poètes vagissent et que les artistes modernes sont en attente de jugement, pendant que les fils de riches s'instruisent et que les classes pauvres se résignent dans l'attente d'un Rédempteur, le peuple du Québec, en 1937-1938, s'accroit de toutes parts et le clergé catholique fonde de nouvelles paroisses, donnant ainsi a cette nation et a ce pays incertain, cohésion, certitudes et structures, en l'absence d'un État véritable " (article cité en bibliographie, p. 170). On remarquera qu'Orphée participe à plusieurs dimensions du récit puisqu'il est tout à la fois un bourgeois, un poète, un fils de riches et un artiste moderne en attente de jugement. 
convoquée par une série de poèmes, c'est-à-dire de textes versifiés, qui traversent le roman et participent chacun de façon différente à la fiction. Poèmes d'Alfred Garneau, de SaintDenys Garneau, de l'Écossais Robert Burns, d'Apollinaire ou d'auteurs non identifiés s'incorporent ici et là au récit ${ }^{3}$. Liée étroitement à la figure de l'éloquence religieuse et profane sermons et discours ponctuent, en effet, la trame narrative du roman et font écho aux textes versifiés -, l'utilisation discursive de ces textes poétiques vient, en quelque sorte, compléter le discours critique qui se dégage de la figure du poète. Bien que Le ciel de Québec développe sans doute à d'autres niveaux sa vision de la poésie, nous nous en tiendrons, dans les limites de cet article, à expliciter ces deux principales représentations qui, pour être manifestes, n'en convoquent pas moins des acteurs et des réseaux figuratifs apparemment assez éloignés d'une réflexion poétique et, plus généralement, littéraire.

$$
\text { *** }
$$

Qu'il soit dénommé Orphée constitue déjà un signe de l'appropriation du personnage de Saint-Denys Garneau par la fiction: en dépit de sa réalité historique, en effet, le récit montre bien sa volonté de récupérer et de remodeler l'écrivain. Le nom choisi n'est d'ailleurs pas vide de sens. Outre le fait qu'il désigne un poète épique, Orphée renvoie surtout à un personnage mythique se trouvant à l'origine d'une grande tradition littéraire. Autant que la référence au poète, l'idée de mythe contenue dans le nom d'Orphée s'avère déterminante en regard de la fiction puisque c'est précisément au mythe de Saint-Denys Garneau (mythe entendu ici au sens de réalité illusoire, idéalisée ou déformée) que choisit de s'en prendre Le ciel de Québec. En attente de jugement pour *boursouflure de renommée» (p. 229), Orphée jouit à tort d'une réputation de grand poète; par son nom, la fiction signale d'entrée de jeu cette dimension mythique que la suite du récit se donne pour objet de démonter. L'entreprise de démythification s'avère donc, en quelque sorte, toute entière contenue dans le nom du

3 Précisons que ce sont davantage le rôle des différents poèmes dans la fiction, plutót que les sources intertextuelles elles-mémes, que nous examinerons ici. Ferron multipliant les ruses (fausses attributions, citations masquées ou fictives, etc.), le repérage et l'analyse du réseau intertextuel du Ciel de Québec déborderait certainement les limites d'un article. Pour avoir une idée de la stratégie intertextuelle de Ferron, nous renvoyons a l'article de Guy Monette cité en bibliographie. 
poète. En le privant de son patronyme véritable, le récit se trouve à récuser, d'autre part, non seulement la légitimité du patronyme nobiliaire (de Saint-Denys), mais aussi le droit du poète à porter le nom des Garneau, en l'occurrence celui de l'historien François-Xavier Garneau. L'imposition d'un nom fictif et le choix du substitut renferment déjà, en somme, l'essentiel du * destin » narratif et du contenu thématique du personnage.

L'organisation onomastique forme souvent chez Ferron un système signifiant. Quand un personnage est dénommé (ce qui est assez rare, surtout dans les contes où les acteurs sont plutôt désignés par leur rôle social: le médecin, le curé, la sage-femme, la mère, etc.), son nom contient, règle générale, presque toute la substance de son "périple" narratif ou de ses attributs modaux. L'autre personnage du roman à porter un nom particulièrement significatif, Frank-Anacharcis Scot, assume un parcours opposé à celui d'Orphée, lequel permet justement de préciser la portée exacte de l'échec narratif du poète. S'il renvoie, bien sûr, au traducteur et poète Frank Scott, ancien doyen de la Faculté de droit de l'université McGill (comme le précise d'ailleurs le tableau des principaux personnages historiques annexé au roman ${ }^{4}$ ), Frank-Anacharcis renvoie également au philosophe scythe, Anacharsis (v. $\mathrm{VI}^{\mathrm{e}}$ siècle), qui de retour dans sa patrie fut tué pour impiété: missionnaire auprès des Esquimaux, le fils du Bishop Dugald Scot reviendra à Québec parce quỉl ne croit plus au Dieu de paix qu'il prêchait jusque-là; s'il ne meurt pas, Frank Scot sera néanmoins mis à la porte de la maison paternelle et choisira de «s'enquébecquoiser $*$. Tout comme Orphée (SaintDenys Garneau) qui, en descendant aux enfers, se conforme au mythe évoqué par son nom, Frank-Anacharcis assume donc, lui aussi, la destinée historique de son homonyme.

L'opposition qui lie Orphée à Frank Scot se manifeste à plusieurs niveaux. Le nom d'origine mythique assigné au poète, par exemple, vient s'opposer au patronyme doublement historique du pasteur (Frank Scott et Anacharsis), comme si le caractère authentique de l'un faisait ressortir encore davantage le statut illusoire et mystifiant de l'autre. L'engagement de Frank-Anacharcis Scot souligne aussi le complet détachement social qui caractérise le personnage d'Orphée. S’il change

4 Ce tableau préparé par Jean Marcel apparaft seulement dans l'édition parue chez VLB éditeur. 
d'objet, l'engagement de Frank ne se dément pas tout au long du récit: tantôt missionnaire chez les Esquimaux, plus tard chargé par Mgr Camille d'aider à la fondation de la paroisse de Sainte-Eulalie, Frank participe à la réalité sociale et historique de son époque. Orphée, au contraire, préfêre la solitude du Manoir à la réalité immédiate du monde ${ }^{5}$. L'échec du poète et, du même coup, de l'expérience poétique qu'il représente réside toutefois principalement dans le contraste qui oppose les parcours narratifs des deux personnages. Alors que Frank-Anacharcis «s'enquébecquoisera par le bon bout", en faisant l'amour avec une prostituée à l'Hôtel des Voyageurs, Orphée passera, quant à lui, à côté de l'amour charnel en échouant dans sa tentative pour ramener Eurydice des enfers. Une fois réalisée la première étape de son assimilation au pays québécois, Frank-Anacharcis (devenu François) accèdera à l'écriture. Le chapitre de conclusion le révèle, en effet, narrateur du récit et auteur d'une future chronique intitulée: La vie, la passion et la mort de Rédempteur Fauché. Si l'accession à la vie québécoise et à la parole (François, dans la conclusion, s'exprime à la première personne) vient clore le parcours de François-Anacharcis, c'est, au contraire, la mort de la parole et du poète qui termine le périple d'Orphée aux enfers: «- Eurydice, lui dis-je, tu m'as aimé et je t'ai fui, t'outrageant d'une irrémédiable façon: tu en es morte. J'ai compris alors que je t'aimais, que ta mort était la mienne et que sans toi, je survivrais sans vie» (p. 384). Bien que Mgr Camille conseille à Orphée de continuer à écrire et lui prédise même qu'il sera bientôt un grand poète, la messe en noir célébrée par le prélat à l'intention du jeune homme symbolise clairement la mort définitive des mots : "Orphée n'ose répondre [...] qu'il ne croit plus guère à ce qu'il voit, encore moins à ce qu'il dit, et que l'opacité fondamentale des choses

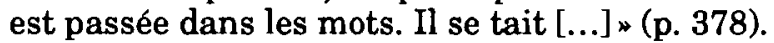

En faisant se dérouler dans le même lieu, c'est-à-dire à l'Hôtel des Voyageurs, bordel de la basse-ville de Québec, la descente aux enfers d'Orphée et l'enquébecquoisement de François-Anacharcis, la fiction rapproche le destin des deux hommes. Elle force encore davantage le rapprochement en faisant, dans chacun des cas, une véritable épreuve initia-

5 En cela, le fils contraste aussi avec sa mère, Calliope, devenue communiste (son engagement, toutefois, se limite a porter un regard theorique sur le réel). Voir à la page 95 du roman. 
tique de la rencontre avec une femme. C'est, en effet, l'union physique avec la prostituée Georgette qui permet à François d'accéder à la nationalité québécoise, comme c'est l'incapacité d'en faire autant avec Eurydice qui condamne définitivement Orphée à la solitude. Si l'échec de ce dernier réside, comme le veut d'ailleurs le mythe, dans le fait de s'être retourné, il réside également dans son indécision et ses scrupules à prendre Eurydice sur le plancher du bordel " comme une putain» tel qu'elle l'y invite (p. 385). L'épisode de la descente aux enfers montre à quel point Orphée hésite à répondre à l'appel d'Eurydice:

- Orphée, tu es venu me chercher aux enfers, je t’ai suivi, je suis là derrière toi, prête à remonter au jour dès que tu daigneras y remonter. J'entends rire là-haut. C'est la vie qui nous appelle. N'hésite plus [...]. - Eurydice, je t'aime, mais je reste plein d'appréhension. - Douterais-tu de moi, Orphée? - Si seulement je pouvais te voir avant de monter! - C'est défendu, Orphée, tu le sais. [...] - Attendons encore un peu. [...] - Continue, mon doux sauveur, vite monte qu'enfin revenue au jour, je tombe dans tes bras, que tu me prennes et tu m'embrasses [...] - Eurydice, en haut, c'est le bordel. - Pour une fois tu me prendras comme une putain. Eurydice! (p. 384-385)

C'est finalement parce qu'il refuse l'amour charnel, symbole de la vie, que le jeune Orphée du Ciel de Québec perd son Eurydice, c'est-à-dire tout à la fois l'amour, la vie et l'écriture. Le mythe de la descente aux enfers permet donc de lier la mort de l'écriture au refus de l'amour humain.

La fiction développée dans Le ciel de Québec unit par un rapport essentiel la parole à la réalité immédiate du monde. La réussite de François-Anacharcis montre bien que pour accéder à la parole, il faut d'abord accepter la vie dans ses manifestations les plus concrètes: l'amour de la femme, comme celui du pays ${ }^{6}$. La défaite d'Orphée illustre, pour sa part, que le refus de l'amour détruit la possibilité même de l'écriture. L'exil en soi-même auquel se condamne Orphée entraîne la mort des mots. Si Orphée (Saint-Denys Garneau)

6 Lors de sa tournée d'évangélisation, Frank-Anacharcis manquera justement l'occasion de coucher avec une Esquimaude; la tradition d'offrir une femme à leur hóte étant, semble-t-il, disparue (p. 118). Déçu de son expérience missionnaire, il reviendra donc sans avoir pu s'intégrer à la communauté. 
26

forme ici le modèle du faux poète, jouissant indûment d'une boursouflure de renommée, ce n'est donc pas parce que sa poésie n'est pas patriotique mais parce qu'elle exclut une expérience concrète du réel - aussi bien du pays que de la femme - et ne constitue pas, de ce fait, une parole vivante et enracinée. La réussite de François-Anacharcis vient justement expliciter les causes et la profondeur de l'échec d'Orphée.

\section{*** *}

L'échec narratif d'Orphée et la réussite de Frank constituent finalement un point de vue sur l'écriture. Plus encore que les propos du narrateur, la trame fictionnelle s'attache à démontrer les fondements de l'impasse poétique d'Orphée. L'anecdote propose, en somme, à travers les parcours opposés des deux personnages, une version narrative de sa critique et de sa condamnation de Regards et jeux dans l'espace de SaintDenys Garneau. Sans même s'en prendre aux valeurs spirituelles et au mysticisme reconnu de l'écrivain (le roman ne cite que trois poèmes de Garneau et ne propose nulle part une véritable analyse de l'œuvre; les commentaires du narrateur s'attachent, en fait, davantage à la biographie de l'auteur), le texte réussit donc à problématiser l'échec poétique de l'écrivain. Le point de vue défendu par Le ciel de Québec ne réside pas tout entier, cependant, dans la diégèse. Le roman recourt aussi à toute une série de discours, de sermons et de poèmes qu'il organise en un système précis d'oppositions, lequel reprend et prolonge au niveau discursif la polémique engagée jusque-là au niveau fictionnel.

Le ciel de Québec entrecoupe son récit d'assez longs discours. La visite des prélats de Québec au petit village des Chiquettes, surtout, multiplie les morceaux d'éloquence: le chef du village, le cardinal et la vieille capitainesse prennent à tour de rôle la parole, la harangue de la capitainesse recouvrant même un chapitre entier du roman (le chapitre $\mathrm{X}$ ). Si ces discours ajoutent peu, semble-t-il, à l'action du récit, ils entretiennent toutefois des rapports certains avec les quelques textes versifiés qui traversent l'ouvrage. Les poèmes d'Alfred Garneau et de Saint-Denys Garneau, en particulier, trouvent dans les envolées oratoires du cardinal et de la capitainesse leur exacte contre-partie ${ }^{7}$. Manifestement construites pour

7 Pour des raisons d'économie d'espace, nous ne pourrons rendre compte de tous les discours et poèmes contenus dans le roman. 
s'opposer à la poésie des Garneau, ces démonstrations d'éloquence constituent tout à la fois une critique et une réecriture des poèmes retenus par le narrateur.

Bien qu'elle s'adresse aux trois prélats, la longue oraison de la capitainesse Eulalie interpelle en premier lieu le poème de Saint-Denys Garneau cité au chapitre XXX du roman. Faisant état de sa profonde humiliation, celle de toutes les nations amérindiennes, le discours de la vieille Eulalie fait très précisément écho au texte du jeune Orphée dans lequel celui-ci exprime, lui aussi, sa souffrance. Au «C'est eux qui m'ont tué / Sont tombés sur mon dos avec leurs armes / Mont tué / Sont tombés sur mon cœur avec leur haine / M'ont tué [...]" (p. 325), le discours d'Eulalie vient opposer la blessure ancestrale du peuple amérindien ( $[$ [...] après toute une vie à recevoir des coups de gens distraits qui ne remarquent même plus qu'ils vous frappent " p. 78); bien plus, au caractère accusateur du poème, la harangue d'Eulalie oppose plutôt un désir de pardon et de réconciliation ("vous saurez dorénavant, grands princes, toutes nos haines et toutes nos souffrances: ne les oubliez plus même si nous les avons déjà oubliées " p. 79). Parfaite antithèse du poème de Saint-Denys Garneau, le discours de la capitainesse en constitue par le fait même une critique rigoureuse. L'accueil respectueux et admiratif que lui réservent les prélats de Québec ajoute par ailleurs à la disgrâce du poème: le succès du discours implique inévitablement le rejet de son contraire.

Le discours prononcé par le cardinal s'oppose tout aussi nettement, pour sa part, au poème d'Alfred Garneau cité au chapitre XXX du roman. Si l'oraison du cardinal et le poème de Garneau s'adressent tous deux au pauvre et à l'illettré, le premier choisit de lui rendre hommage et de lui témoigner un respect sincère ( -Moïse Chrétien, je suis ému; votre éloquence m'a touché. Si vous dites ce que pensent vos nations et votre peuple, en vérité, en vérité, je ne connais pas de village plus catholique que le vôtre» p. 71), le second préférant plutôt l'admonester et le couvrir de sa condescendance ( Malheur au pauvre aigri qui de sa lèvre torse / Où flotte une écume de fiel, / Insulte à la justice, à l'amour, à la force / De ce Dieu qui créa le ciel!» p. 327). L'ìmage honorable des citoyens du village des Chiquettes, leur sens de l'accueil chaleureux et l'éloquence de leur chef forment, par rapport au portrait pitoyable dressé par Alfred Garneau, un contraste 
pour le moins aigu. À nouveau le texte oppose au poème par le biais, cette fois, du discours du cardinal - sa critique et son contre-exemple 8 .

"Version revue et corrigée» de la poésie des Garneau, les discours du cardinal et de la vieille Eulalie portent un jugement esthétique sévère à son endroit. Non content de conduire Orphée à une mort symbolique, le texte ajoute à l'impasse narrative du poète la disqualification discursive de ses écrits (la poésie d'Alfred Garneau étant, bien sûr, associée à celle de son petit-fils). Bien que l'action entreprise au niveau fictionnel se construise autrement au niveau discursif (dans le premier cas ce sont des parcours narratifs qui s'opposent, dans le second des unités textuelles), elle vise le même but: démontrer que l'expérience poétique de Saint-Denys Garneau ne mérite pas le respect et la reconnaissance qu'on lui porte.

$$
\text { *** }
$$

L'échec de Saint-Denys Garneau s'étendrait-il à toute écriture poétique? Aucun autre poète, rappelons-le, ne semble devoir former la contrepartie positive d'Orphée dans Le ciel de Québec: si le roman s'achève sur la naissance d'un écrivain, François-Anacharcis s'adonne à l'écriture d'une chronique et ce sont des discours qui viennent faire la leçon aux poèmes. Est-ce à dire que la poésie ne peut constituer une parole vivante, qu'elle se réalise essentiellement en dehors de la réalité immédiate du monde, de l'amour et du pays, qu'elle ne rencontre pas les exigences énoncées et défendues par le roman? Ce serait ignorer toutefois un fait textuel important: que pour dépeindre l'enquébecquoisement de Frank-Anacharcis, le texte ait eu justement recours au truchement d'un poème.

L'acte sexuel par lequel Frank-Anacharcis accède à la nationalité québécoise n'est pas véritablement narré. C'est plutôt un texte versifié qui en tient lieu ${ }^{9}$. Que l'initiation charnelle, condition première pour atteindre la parole,

8 On remarque, d'autre part, que le narrateur interrompt à maintes reprises le poème de Garneau pour le ridiculiser, alors que le discours du cardinal est entrecoupe, pour sa part, des réflexions personnelles de $\mathrm{M}^{\mathrm{g}}$ Camille, heureux d'en apprécier les qualités rhétoriques.

9 Selon toute vraisemblance, ce poeme serait de Ferron. Qu'il puisse s'agir d'un poème d'un autre écrivain ne changerait de toute façon rien au róle et à la signification qu'il assume dans le roman. 
apparaisse dans le récit sous la forme d'un poème, montre bien que l'expérience poétique peut constituer une parole vivante et prendre racine dans la chair et l'amour. La peur de l'amour charnel ayant conduit Orphée à l'échec et au silence trouve, dans ce poème, une éclatante riposte:

Vois de tout mon corps l'arc obscène

Tendre à se rompre pour darder

Comme son trait le plus infâme

Implacablement au ciel l'âme

Que mon sein ne peut plus garder (p. 363).

"[...] car tout a commencé par le Verbe et le Verbe s'est fait chair pour prendre bouche et mieux s'exprimer...» (p. 71) affirme le cardinal dans son discours aux Chiquettes, frôlant là l'hérésie. Le ciel de Québec prolongera justement l'audacieuse interprétation de la parole biblique esquissée par le cardinal et proposera, par ce dernier poème, la manifestation exemplaire d'un verbe qui parvient vraiment à se faire chair. L'épreuve ultime de François-Anacharcis, celle par laquelle il atteint enfin la nationalité québécoise, cette victoire finale est donc aussi celle de la poésie: poésie de chair toutefois qui, renvoyant "implacablement au ciel l'âme " que son " sein ne peut plus garder ", choisit l'amour d'Eurydice et donne clairement son congé au mysticisme d'Orphée de Saint-Denys Garneau.

\section{Bibliographie}

Ferron, Jacques, Le ciel de Québec, Montréal, VLB éditeur, 1979, $408 \mathrm{p}$.

Le Blanc, Alonzo, *Le Ciel de Québec, roman de Jacques Ferron », Dictionnaire des ouvres littéraires du Québec, sous la direction de M. Lemire, Montréal, Fides, 1984, tome IV, p. 170-175.

Monette, Guy. «Les poètes de la Confédération dans Les confitures de coings de Jacques Ferron , Voix \& images, vol. VIII, $n^{\circ} 3$, printemps 1983, p. 421-426.

Smith, Donald, «Jacques Ferron et les écrivains», Voix \& images, vol. VIII, $n^{\circ} 3$, printemps 1983, p. 437-453. 